\title{
Self-Sustained Debacle Repression Using Zig-Bee Communication
}

\author{
Mr. Kaustubh Patil, Promod Kakade \\ Department of Computer Engineering, V.I.I.T Pune India \\ kaustubhudaypatil@gmail.com
}

\begin{tabular}{|l|l|}
\hline Article History & \multicolumn{1}{c|}{ Abstract } \\
$\begin{array}{l}\text { Article Submission } \\
22 \text { June } 2014 \\
\text { Revised Submission } \\
7 \text { September 2014 } \\
\text { Article Accepted } \\
28 \text { November 2014 } \\
\text { Article Published } \\
3 I^{\text {st }} \text { December 2014 }\end{array}$ & $\begin{array}{l}\text { If an accident happens, nature of the humans is to criticize somebody else instead } \\
\text { understanding the mistake. Finally, European study says that in } 80 \% \text { of vehicle } \\
\text { accidents especially motor vehicles, drivers criticize the other person instead of } \\
\text { For any accident to happen, major cause is speed of the driver. So, we should go for } \\
\text { new methods to eradicate such type of problems. Assessment of accidents are done } \\
\text { to identify the reason or series of accidents that are occurred and to avoid fresh } \\
\text { accidents of the same type. So, we are going for Self-sustained Debacle Repression } \\
\text { method identified by the combination of different independent solutions. An SDR } \\
\text { development shape tomorrow's safe. It also comes with collision avoidance } \\
\text { Mechanisms such as: Avoiding the collision using Ultrasonic Sensor and ARM } \\
\text { processor and Collision Indication (car-to-Xs Communication) using ZIGBEE } \\
\text { technology. } \\
\text { Keywords: Collision avoidance, vehicle safety, obstacle detection, sensing, safety } \\
\text { communication. }\end{array}$ \\
\hline
\end{tabular}

\section{Introduction}

WHO has provided Road Safety status report globally says that 1.3 million humans die because of road accidents and India tops the chart. Inside India, Maharashtra \& Andhra Pradesh leads with 1, 37,000 deaths, accompanied by UP with 1, 25,000 deaths. In recent years, many research studies have identified different features of road safety. They are as follows. Accidents because of drunken persons, Risk of the driver due to Alcohol effect, provoking the drivers to be safe, employing air bags in cars, using seat belts in cars etc.

Tariff systems have improved which is an important aspect for vehicles on the ground in the last decade. Accidents can happen in many different ways. Car accidents are one of the most harmful situations that happen every day in society. Speaking practically, accidents which are occurred are not considered as accidents: crashes that may be eliminated. There are four important aspects that come up with accidents. They are Equipment Failure, Roadway Design, Poor Roadway Maintenance and Driver behavior. This analysis is shown in fig 1.

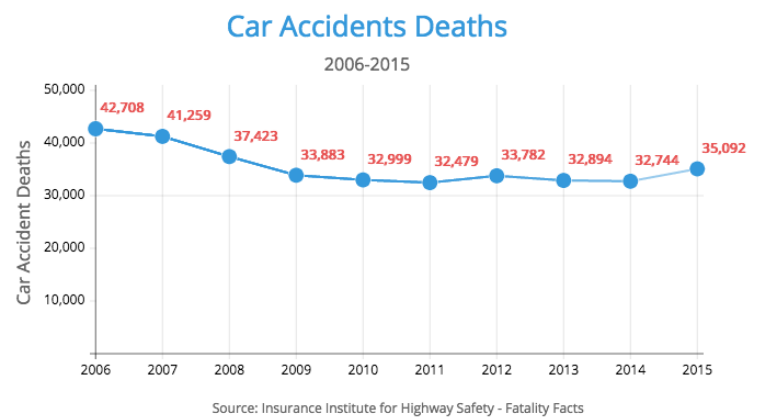

Fig 1: Accident rate analysis 


\section{Related Work}

Accidents that are happened in which thirty percent are due to the over speed. Almost 12,628 were dead because of uncontrollable speed of the vehicle. Driver will be aware of the speed but not about danger that is going to happen. Over speed is not only the cause for accidents. Also accident occurs, if the speed crosses at least by 5 $\mathrm{mph}$ in mistaken location is dangerous. So, the government has been taking actions to safe journey by warning about allowable speeds on the roads especially on NH. Still, speed is allowing the accidents to happen. Hence, we are discussing about the methods employed to decrease accidents in the traffic.

Advancement of automobile industry tends towards employing smart technology to avoid some collisions like injuries. The electronic sensors give the benefit of complex collision identifying methods. They are also adopted simply in the automobile system. Employing electronic sensors are becoming more and more in real-time because of sophisticated embedded systems. Here, we design a mobile robot that is controlled by a mediator for the safety of humans. To reduce the number of accidents we propose SDR technique which aims to provide the prior knowledge regarding the forthcoming collision to the user and to act wisely according to the nature of the collision for safe driving.

\section{SDR System Configuration}

SDR is an important collision avoidance system that contains sensors. It also has a transceiver for the communication purpose which is placed in the vehicle to alert the driver if any danger might occur in front of the road. Sensors will identify car's closeness to the other vehicle on the road and when it senses the speed when moving around the arc. Sensors also transmit and receive signals from traffic lights, disturbances on the road etc. Based on the embedded system employed, it alerts the vehicle owner or the driver to put in brake. The block diagram of SDR Transmitter and Receiver are shown in figure 2 and figure 3 respectively.

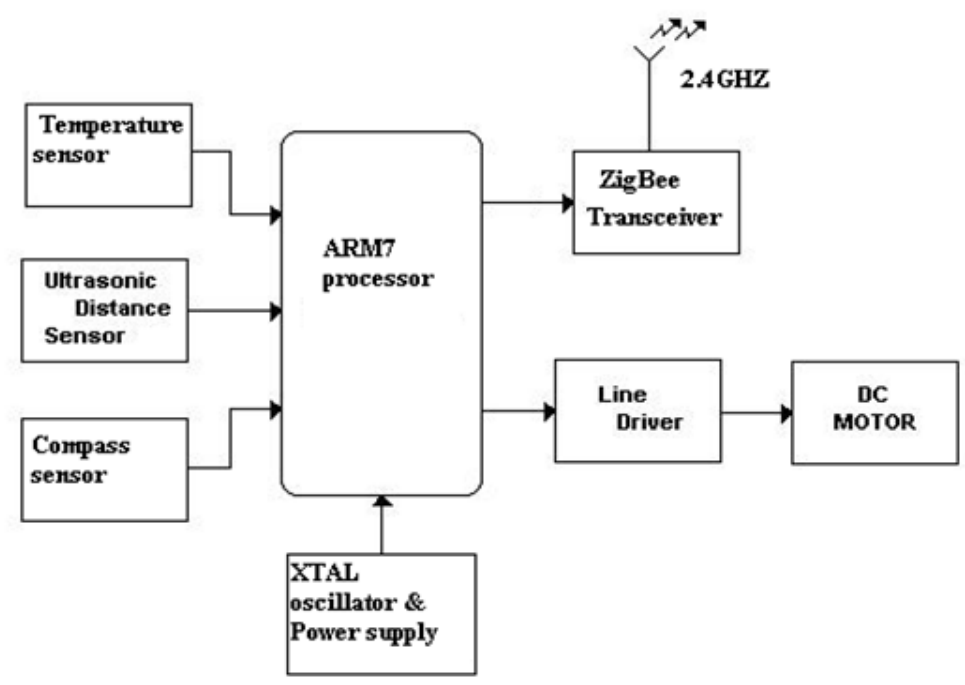

Fig 2: block diagram of SDR Transmitter

In the world of embedded-processor systems, cost and power consumption are to be noted widely. These are the commanding aspects in embedded systems where it is of low cost and small sized microprocessors and microcontrollers employed. RISC instruction set architecture is generally a 32-bit ARM. These processors were suitable for low power applications. The ARM architecture provides many different additions regarding the functionalities (technologies) to expand the "normal" RISC features. It is a widely used architecture. ARM7 is a microprocessor having 32-bit RISC consuming less power which is employed in low cost, power applications. The family of ARM7 includes 16-bit instruction set at a cost of 8/16-bit system and empower the performance of 32-bit. 


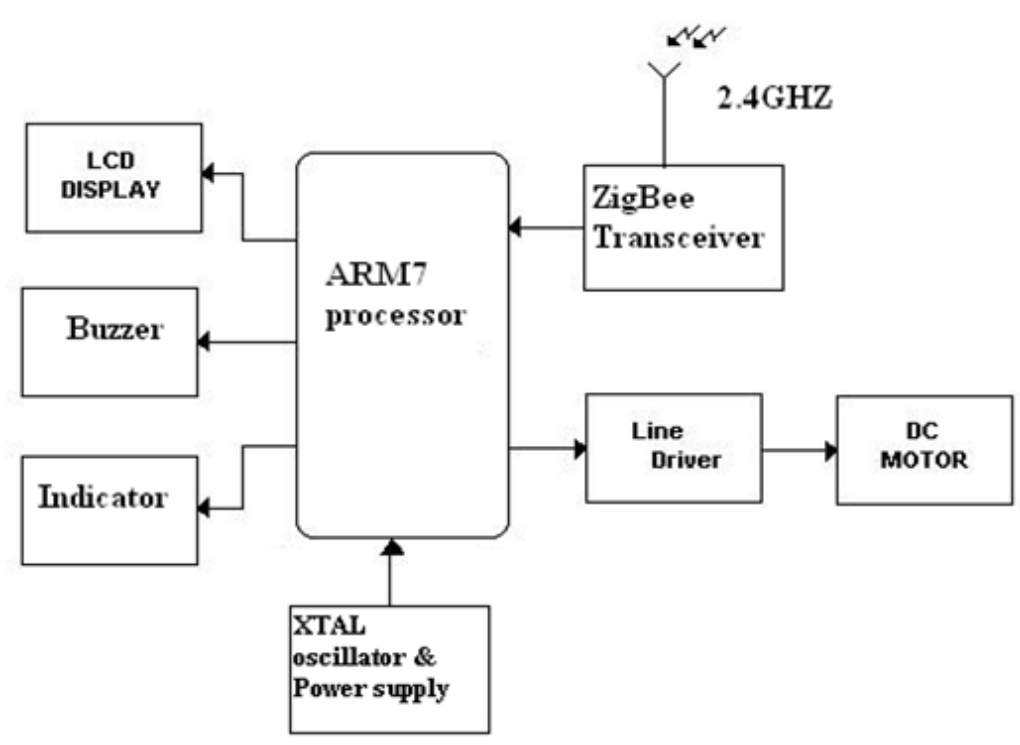

Fig 3: block diagram of SDR Receiver

Here, in this technique employing sensors in evaluating distance avoids the adoption of high cost transducers and difficult DSP techniques. The main purpose for going to ultrasonic sensors is the cost which is low, flexible in design, simple to employ and identifies location of the object inside $10 \mathrm{~m}$ range. In order to avoid collision from other vehicle ahead on the road, the main important aspect is to identify the distance between vehicle and hurdle in front. Also, speed of the vehicle whether it is accelerated or decelerated is identified. Hence, a brake is applied. Initiation of automatic brake is applied when the vehicle distance to the obstacle is very small. Ultrasonic sensor is the one which emits an electromagnetic wave and senses the wave that is reverted back. Operating frequencies of ultrasonic sensors are ranging from $20 \mathrm{kHz}$ to $20 \mathrm{MHz}$ based on the signal travelling in the medium like gas, liquid.

If an ultrasonic pulse wave is hit by an obstacle, small portion or whole pulse might get reverted back to the transmitter which is known as echo. Also, it is identified by the path of the receiver. So, if we identify the travel time of forward pulse and reverse echo, distance can be calculated. Actually, ultrasonic sensors are employed to identify the distance of the vehicle from the obstacle ahead of the sensor. So, we employ these sensors in systems which necessitate calculation of distances.

\section{Simulation Results}

Advancements in avoiding cables adding sensors to processors are increasing due to the employment of many sensors in cars. So, we are going for a wireless technology such as Zig-Bee technology. Intra-car wireless sensor network is happening only because of Zig-Bee. Zig-Bee Technology is projected so that it minimizes the consumption of energy. This allows the long lifetime of device in the case of accumulator source using. The additional advantage of Zig-Bee technology in comparison with another communication forms is that devices in network can direct the data with each other and can be mobile. Moreover the devices do not need to be in direct range of device with which it wants to communicate. The technology is achieved to assure reliable transmission of data across very noise environments. Nowadays the security mechanisms are used as support for safety-related wireless machine, which communicate across Zig-Bee and other wireless media. Increasing population around the globe, Zig-Bee operates in $2.4 \mathrm{GHz}$ band to distribute a reliable and simple standard around the globe.

The ultrasonic sensor tracks the path of the vehicle and the compass sensors give the direction of the car, as soon as the car sense the obstacle through sensors it will alert/warn the driver with buzzer sound and displays the 
corresponding message in the LCD display. If the driver does not take necessary action within the specified time, then the speed of the car is reduced gradually. If a car is under a sudden breakdown and that can be transmitted to the neighboring vehicles through the message as "THERE IS A VEHICLE BREAKDOWN, PLEASE DRIVE CAREFULLY" in the LCD display with the buzzer sound. The system model is shown in figure 4 and flowchart is shown in figure 5 .

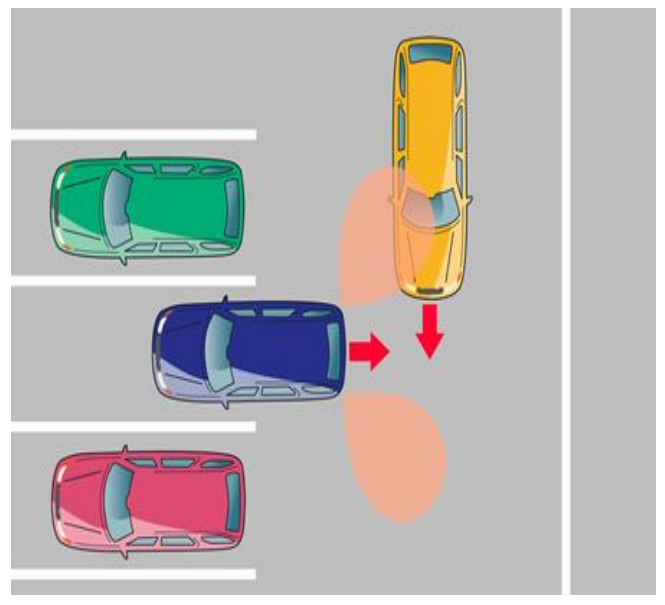

Fig 4: Proposed system model

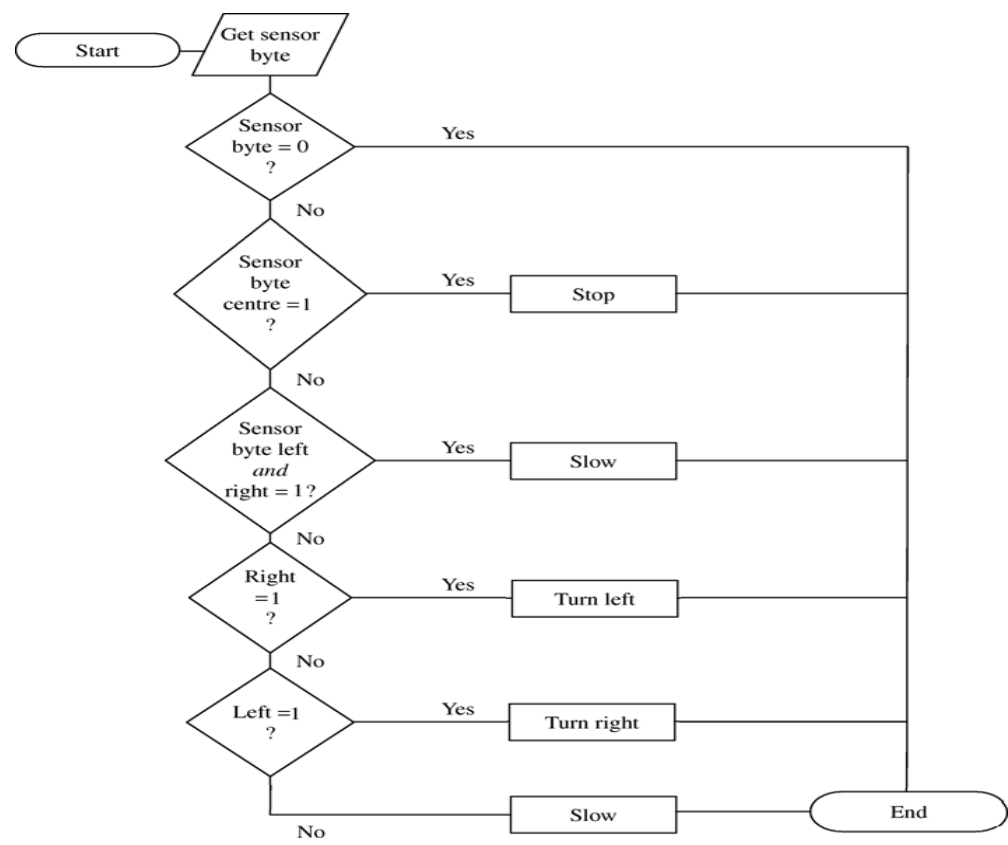

Fig 5: Flowchart Representation of proposed system

Figure 6 shows the simulink model of the proposed system. The rate of obstacle and Graph 2 shows the motor velocity. Whenever the obstacle occur the movement of the car in the direction of obstacle should be avoided and so the motor velocity is reduced correspondingly. Based on this concept we have simulated our program. As the rate of obstacle increases the motor velocity is reduced and if the rate of obstacle decreases the velocity of motor is increased. 


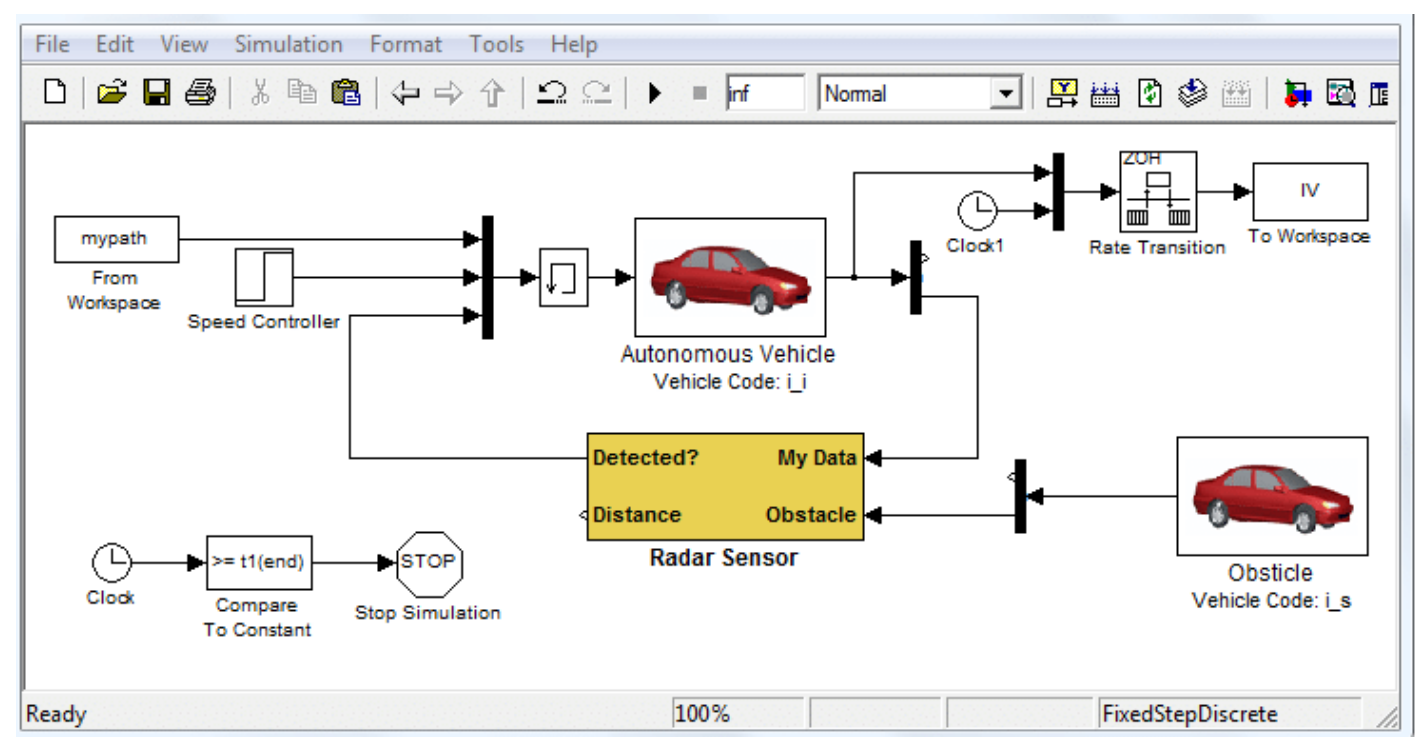

Fig 6: Simulink Simulation of proposed system

\section{Conclusion}

The action of controlling motor speed is performed automatically depending on the obstacle distance from the car. Whatever the speed of the vehicle may be and once if the obstacle is sensed the process of communication to other vehicles is done within $3 \mathrm{mS}$. Numerous obstacle detection algorithms for autonomous driving have been proposed to avoid the unnecessary threat to human life. The possibilities of accident reduction have been discussed in this paper. Based on the above results, we are now on the process of developing our hardware kit which will provide pleasure driving with a better environment.

\section{References}

[1] David, Flach.A(2010),"Car-2-X and Pedestrian Safety”, Vehicular Technology Magazine,IEEE, Vol5,pp.70-76.

[2] Chisalita.I, Shahmehri.N (2007)," On the Design of Safety communication Systems for Vehicles", IEEE Spectrum, Vol-37, pp.933-945.

[3] Gallagher.B, Akatsuka.H, Suzuki. H(2006),"Wireless Communication for Vehicle Safety”,Vehicular Technology Magazine,IEEE,Vol-1,pp.4-24.

[4] Kashif Dar, Mohamed Bakhouya, Jaafar Gaber, and Maxime Wack(2010),"Wireless Communication Technologies for ITS Applications", Communications Magazine, IEEE, Vol-48,pp.156-162.

[5] S. Hu, C. Lin and Y. Tseng, "Automatic parameter selection for the ZigBee distributed address assignment mechanism," 2013 IEEE 24th Annual International Symposium on Personal, Indoor, and Mobile Radio Communications (PIMRC), London, 2013, pp. 2062-2066, doi: 10.1109/PIMRC.2013.6666483.

[6] M. Fang, J. Wan, and X. Xu. A Preemptive Distributed Address Assignment Mechanism Wireless Sensor Networks. In Proc. of WiCOM'08. 4th International Conference on Wireless Communications, Networking and Mobile Computing, pages 1-5, 2008.

[7] S.-C. Hu, Y.-C. Wang, C.-Y. Huang, and Y.-C. Tseng. Measuring air quality in city areas by vehicular wireless sensor networks. Journal of Systems and Software, 84(11):2005-2012, 2011.

[8] M. Garcia, S. Sendra, J. Lloret, and A. Canovas. Saving energy and improving communications using cooperative group-based wireless sensor networks. Telecommunication Systems, 2011.

[9] X.-M. Huang and J. Ma. Optimal distance geographic routing for energy efficient wireless sensor networks. International Journal of Ad Hoc and Ubiquitous Computing, 1(4):203-209, 2006. 
[10] H. S. Kim and J. Yoon. Hybrid distributed stochastic addressing scheme for ZigBee/IEEE 802.15.4 wireless sensor networks. ETRI Journal, 33(5):704-711, 2011.

[11] M. Kochhal, L. Schwiebert, and S. Gupta. Role-based hierarchical self-organization for wireless ad hoc sensor networks. In Proc. of ACM Int'l Workshop on Wireless Sensor Networks and Applications (WSNA), 2003.

[12] S. Kulkarni, A. Iyer, and C. Rosenberg. An Address-Light, Integrated MAC and Routing Protocol for Wireless Sensor Networks. IEEE/ACM Trans. on Networking, 14(4):793-806, 2006. 